\title{
De la orden de las hermanas agustinas a la enfermeria contemporánea
}

\section{From the order of Augustinians' Sisters to the contemporary nursing}

\author{
Rodolfo Rodríguez Gómez
}

Forma de citar: Rodríguez Gómez R. De la orden de las hermanas agustinas a la enfermería contemporánea. Rev Univ Ind Santander Salud. 2016; 48(4): 554-560. DOI: http://dx.doi.org/10.18273/revsal.v48n4-2016014 @c) (1)

\section{RESUMEN}

La historia de la enfermería ha estado llena de apasionantes eventos que han hecho de esta profesión un elemento clave en el desarrollo de la sociedad. En medio de ese viaje, un aspecto esencial para la ciencia del cuidado fue el papel llevado a cabo por diferentes órdenes religiosas durante la Edad Media, especialmente el de la orden de las Hermanas Agustinas. Desde entonces, la vocación de grandes personalidades en la actividad asistencial gestó el arte del cuidado como profesión. En esta historia se ha forjado una labor que afronta grandes retos tanto en la práctica como en lo epistemológico. Dicho camino se ha labrado con dignidad, sacrificio y constantes desafíos para lograr un espacio profesional respetable. Hoy en día, gracias a la consagración de aquellas mujeres y quienes a diario honran su memoria, la enfermería se posiciona como protagonista ante las nuevas y complejas dinámicas del campo de la salud.

Palabras clave: Historia, enfermería, hospitales, salud, enfermeros.

\begin{abstract}
The history of nursing has been full of exciting events that have made this profession a key element in the development of society. Amid of these important events, an essential aspect for science was the role carried out by different religious orders during the Middle Age, especially the Order of the Augustinian Sisters. Since then, the vocation of great personalities in the care activity conceived the art of care as a profession. In this story it has forged the way of a labor that faces major challenges both in practice and in the epistemological part to achieve a respectable professional space. This path has been carved with dignity, sacrifice, and constant challenges to achieve a respectable professional space. Today, thanks to the dedication of those women and who daily honor their memory, nursing ranks as protagonist with enormous potential to new and complex dynamics of the field of health.
\end{abstract}

Keywords: History, nursing, hospitals, health, nurses.

\footnotetext{
1. Diagnóstico Ocular del Country. Bogotá. Colombia Correspondencia: Rodolfo Rodríguez Gómez. Dirección: Calle 22 A N 83 - 10 Bogotá. Correo electrónico: fitopolux@hotmail.com. Teléfono: 3124288819
} 


\section{INTRODUCCIÓN}

La travesía histórica de la enfermería ha sido convulsa y retadora, pero al mismo tiempo fascinante. El ejercicio del arte de los cuidados que en un principio fue ejercida por hombres de manera intuitiva y posteriormente por mujeres, ha sido una actividad fundamental para la preservación de los seres humanos ${ }^{1}$. No obstante, el discurrir de la enfermería desde sus inicios hasta lograr su estatus profesional, ha estado enmarcado por épocas sociales revolucionarias donde se ha destacado la tenacidad de sus protagonistas. Sin desconocer el papel del hombre en el cuidado enfermero, las mujeres han ejercido un rol trascendental tanto en el desarrollo de la actividad asistencial como en la construcción social y teórica que ha posicionado a la enfermería como profesión digna. No por nada, se considera que la enfermera es el espejo donde se refleja la situación de la mujer a través de los tiempos ${ }^{2}$. Pero conocer la historia no solo es relevante para reconstruir el pasado, sino también para honrar los orígenes, dar mayor valor al quehacer diario y respetar los símbolos que emergen de aquella construcción social. Para una profesión que le ha costado tanto llegar a ser lo que es, la memoria de quienes han dado la vida misma por lograrlo rescata el valor de épocas pasadas, re-significa el presente y da cabida para reflexionar sobre el futuro.

El presente artículo se basó en una extensa revisión documental que incluyó tanto textos físicos como diferentes bases de datos y recursos electrónicos. La búsqueda se realizó sin restricción en el tiempo y la información se sistematizó de acuerdo al periodo histórico correspondiente. Se utilizó una amplia terminología de búsqueda la cual se ajustó de acuerdo al tema y la fuente primaria a consultar.

\section{La Orden de las Agustinas y el Hôtel-Dieu de París}

A pesar de que la historia del cuidado es milenaria, un hito trascendental en el desarrollo de la enfermería sobresale durante la Edad Media. Se trata de la creación de la Orden de las Agustinas, considerada como una de las más antiguas órdenes religiosas dedicada de forma exclusiva al cuidado de los enfermos ${ }^{3}$. Esta comunidad, pasaba gran parte del tiempo en las salas del hospital ya que tenía a su cargo la admisión y el cuidado de los pacientes, y salía únicamente para visitar a los enfermos en sus domicilios ${ }^{3}$. Estaban bajo el mandato de una Maitresee o Superiora, y debían pasar por tres etapas: Filles en aprobation, cuando eran principiantes; Filles blanches, cuando recibían la túnica blanca; y Filles á chaperón, al recibir la capa negra ${ }^{3}$. Los hospitales episcopales de aquel entonces surgieron por la transformación de los tradicionales xenodochiums, es decir, los hospitales para pobres y peregrinos. ${ }^{4}$. De esta manera, el cristianismo tuvo gran influencia en la filosofía del cuidado de los enfermos los cuales se convertían en el vehículo a través del cual se ponía en práctica la caridad cristiana y con ello, se labraba el camino para la salvación del alma ${ }^{5}$.

En el año 651 d.C., se construyó el Hôtel-Dieu, el que es hoy el más antiguo hospital de París y que fue fundado por el obispo Landerico, posteriormente llamado San Landerico ${ }^{6}$. Las religiosas pertenecientes al HôtelDieu asumieron por entonces la Regla de San Agustín. Por tal motivo, las Hijas de San Cristóbal, pasaron a denominarse Hermanas Agustinas o Hermanas Hospitalarias de San Agustín 6 . En tiempos del obispo Landerico, es decir, en el siglo VII, además de la catedral existía una capilla dedicada a San Cristóbal. Junto a ella, existía un hospital para pobres denominado Hospital de San Cristóbal, el que años después se convertiría en el famoso Hôtel-Dieu que significa casa de Dios $^{7}$. El trabajo de las religiosas en este hospital era principalmente la labor asistencial con los enfermos, cuyo más antiguo registro está documentado en el primer Estatuto del Hôtel-Dieu que data de $1217^{8}$.

En un principio, las Hermanas Agustinas, antes llamadas Hermanas de San Cristóbal, se dedicaban a ciertas labores como el cuidado de la capilla y la ropa $^{9}$. Sin embargo, por orden del obispo, comenzaron a atender a los enfermos del hospital ${ }^{10}$. Durante los siglos XII y XIII, los mejores hospitales se podían encontrar en Francia y el mayor de ellos era, por supuesto, el Hôtel-Dieu de París ${ }^{11}$. No obstante, pese a que se trataba de un renombrado sanatorio de la época, durante toda la Edad Media careció de botica, es decir, de farmacia comunitaria. Fue mucho tiempo después de su fundación cuando se instauró este servicio que era atendido por una hermana profesa, una fille blanche, y un sirviente que tenía la labor de preparar y repartir las pócimas, ungüentos y emplastes ${ }^{12}$. En el hospital, las hermanas en pleno derecho podían acoger nuevas novicias a su cargo, ser responsables de una de las áreas del edificio, y tomar la capucha negra de lino o lana a la que obligaba el Estatuto ${ }^{13}$.

Las órdenes seglares sin lugar a dudas hicieron grandes aportes a la enfermería. Hacia el siglo XII, por ejemplo, aparecieron las Beguinas, una orden seglar que debe el nombre a su fundador, el obispo belga Lambert le Bègue. Esta asociación de mujeres 
cristianas tuvo su origen en Bélgica en el año 1184, y siglos más tarde, se contaba con cerca de doscientas mil Beguinas ${ }^{14}$. Pertenecían en especial a la clase media, aunque algunas eran aristócratas y campesinas que vivían de su trabajo en la artesanía textil, el copiado y miniado de manuscritos, y de su labor como enfermeras en hospitales para pobres ${ }^{15}$. Hacia el siglo $\mathrm{XV}$, se les llamaba beatas y fueron reconocidas por ser grandes viajeras y vivir de las limosnas en los viajes de peregrinación que en ocasiones abarcaban grandes distancias ${ }^{15}$. Su labor asistencial consistía en cuidar a los enfermos en los hospitales, visitar a quienes convalecían en sus domicilios y también servir a los pobres, a los abandonados, a los ancianos y a los huérfanos.

La vida de las mujeres durante la Edad Media era difícil. De no llevar una vida matrimonial o religiosa, quedaban pocos caminos como la prostitución o la servidumbre. No obstante, al adoptar la vida religiosa, una opción digna era el cuidado de los enfermos. De esta manera, nacieron las Hermanas de la Caridad -también llamadas Hijas de la Caridad- cuyas primeras integrantes fueron un grupo de jóvenes que se entrenaron como enfermeras del cuerpo y el alma con Luisa de Marillac de Legras. De la mano de San Vicente de Paúl, Luisa de Marillac fue la fundadora de esta sociedad de vida apostólica conocida en Francia como hermanas grises ${ }^{16}$. El número de mujeres que se acogió a esta cofradía creció rápidamente ya que debido a la represión social, los hospitales se llenaron de apestados, locos, delincuentes y pobres, quienes eran señalados y condenados por la sociedad burguesa de la época como los supuestos culpables de la enfermedad ${ }^{17}$.

\section{La enfermería trasciende los límites medievales}

En el siglo XVI y XVII se fundaron en España varias órdenes y congregaciones dedicadas a la asistencia de pobres y enfermos. De gran relevancia fueron la Congregación de los Enfermeros Obregones, la Orden de San Juan de Dios, y la de los Belemitas. Estas dos últimas de gran importancia en la época hispánica, ya que dieron cimiento al sistema hospitalario en Latinoamérica $^{18}$. En el siglo XVII, el papel del enfermero español Andrés Fernández fue trascendental al recopilar técnicas y conocimientos para la formación de los enfermeros de su congregación ${ }^{19}$. En 1617, bajo el título Instrucción para enfermeros ${ }^{20}$, Fernández publicó uno de los primeros manuales de enfermería dirigido a enfermeros. La segunda edición, tuvo lugar en 1625 con ediciones posteriores en 1664, 1680 y
1728 las cuales llevaban un libro adicional sobre el bien morir $^{21}$. Se trata, entonces, de uno de los textos más antiguos de Europa escrito por un enfermero y dirigido a la formación de hombres en el cuidado al enfermo ${ }^{22}$. Constituye, además, evidencia de la presencia de los hombres en el cuidado enfermero que para la época, representaba una actividad con predominancia femenina y esencialmente, mujeres pertenecientes a órdenes religiosas.

Los cuidados realizados por mujeres religiosas se extendieron al resto de Europa entregándoles hospitales para su gestión y organización. En Alemania, se fundaron hacia 1830 las Diaconisas de Kaiserswerth con el fin de redimir presidiarias, cuyas tareas, entre otras, eran los cuidados en el hospital ${ }^{23}$. Sin embargo, por aquellos años el reformismo religioso tuvo un papel determinante en la historia de la enfermería dado que se gestaron nuevos conceptos sociales que dieron paso a la modernización del rol femenino. En esta línea, en países como Inglaterra, Alemania, Noruega, Dinamarca y Suiza, en cuanto a la filosofía de la actividad asistencial se dieron cambios significativos al emerger nuevas posturas en torno a lo religioso ${ }^{24}$. De esta manera, los hospitales creados para curar y albergar enfermos y desamparados, quedaron sin dirección ya que estaban regidos por la Iglesia católica. Fue en Inglaterra, precisamente, donde a mediados del siglo XIX, se empezó a gestar la profesionalización de la enfermería mucho antes que en países católicos como Francia, España e Italia ${ }^{24}$.

Entre 1500 y 1860 la enfermería atravesó por un pasaje turbulento en su historia. Dado que la concepción reinante era que la enfermería constituía más una ocupación vinculada a la actividad religiosa que a la intelectual, esto limitó su progreso científico. Con el reformismo religioso y la desmotivación por el cuidado a los enfermos, muchas personas no idóneas se dedicaron a la actividad asistencial, motivo por el cual, este periodo se bautizó como la 'época oscura de la enfermería' ${ }^{25}$. Hacia 1819 , el médico británico Benjamin Golding manifestaba que reclutar las personas adecuadas para desempeñar el trabajo de enfermeras era una de las más grandes dificultades de un hospital ${ }^{26}$. De hecho, por aquel entonces, las mujeres respetables o las personas adecuadas, no estaban interesadas en la enfermería hospitalaria, ya que era considerada una labor de baja categoría. Sin embargo, los cambios no se harían esperar y en el Londres del siglo XIX, una serie de reformas motivadas por grandes protagonistas, permitirían hacer de la enfermería una reconocida y respetable profesión. 


\section{Florence Nightingale y otras mujeres protagonistas}

Para 1819 no había escuela de enfermería en Londres. No obstante, para finales de siglo, había 12 hospitales con escuela médica y cada uno contaba con su propia escuela de enfermería. Surgía así la llamada enfermera Nightingale, es decir, una persona preparada y respetable para ejercer la profesión ${ }^{26}$. No por nada, Florence Nightingale consideraba que la enfermería era una vocación que requería sujetos aptos e inteligentes a quienes había que remunerar por la calidad de su servicio $^{27}$. Durante la guerra de Crimea, un conflicto bélico entre el Imperio ruso y el Imperio turco apoyado por Francia e Inglaterra, Florence Nightingale organizó en tiempo record un equipo de enfermeras voluntarias para asistir a los soldados enfermos y heridos ${ }^{28}$. Su experiencia durante esta sangrienta guerra, la hizo reflexionar sobre el tipo de persona que atendía a los enfermos, ya que al visitar los hospitales, encontró que a partir de las horas de la tarde las enfermeras estaban ebrias, la higiene era lamentable, y era común ver charcos y ratas cerca de las camas ${ }^{29}$.

A Florence Nightingale le preocupaba que mujeres provenientes de clases bajas de la sociedad sin ninguna formación consiguieran trabajo para cuidar enfermos ${ }^{29}$. Tras la guerra y motivada por tales inquietudes, Florence estableció en el Hospital Saint Thomas de Londres su escuela de enfermería; la Nightingale Training School. Difería de otras escuelas en que las estudiantes recibían clases teóricas a la par de la experiencia clínica en las salas del hospital y en poco tiempo se hizo mundialmente reconocida ${ }^{30}$. En su libro de 1859, titulado Notas sobre enfermería, Florence destaca lo que llamaba el 'arte de la enfermería', enaltece el valor de la calidad del aire y pone en práctica sus conocimientos de estadística ${ }^{31}$. Dicho libro, no solo encumbra a Florence como una gran teórica de la enfermería, sino que representa un aporte crucial para la historia de la estadística sanitaria, la epidemiología y la enfermería moderna ${ }^{32}$. En honor a los aportes de esta mujer visionaria, el Comité de la Escuela de Enfermería Ferrand del hospital Harper de Detroit dirigido por la profesora Lystra Eggert, elaboró en 1893 el Juramento de enfermería que fue llamado Juramento Florence Nightingale ${ }^{33}$.

En el siglo XIX otros hitos encumbraron la emergente profesión. Ethel Fenwick, por ejemplo, no solo aportó a la profesionalización de las enfermeras, sino también a la reivindicación de la mujer en la sociedad ${ }^{34}$. En Inglaterra, trabajó por la estandarización de la educación en enfermería al ejercer activismo en pro de los derechos de quienes ejercían esta actividad y promovió su agremiación ${ }^{34}$. Fue en Liverpool, precisamente, en 1859, donde surgió con éxito el sistema de Enfermería de Distrito, un programa de enfermería comunitaria dirigido por Mary Robinson, que para 1864, ya había sido replicado en otras ciudades de Inglaterra ${ }^{35}$. Para 1881, la estadounidense Clara Barton, tras regresar de Ginebra, fundó la Cruz Roja Americana ${ }^{36}$ y poco después, en 1899, nacía el Consejo Internacional de Enfermeras, el cual hoy en día congrega más de 130 asociaciones nacionales de enfermería y representa a más de 13 millones de enfermeras alrededor del mundo $^{37}$. Dentro de sus objetivos, está el velar por los intereses de la enfermería y las enfermeras en diferentes aspectos como la autonomía profesional, el fomento de las relaciones entre enfermeras de todo el mundo, el incentivo a la docencia e investigación, entre otras.

La transición al siglo XX fue trascendental para la enfermería en las Américas. En este contexto, Linda Richards tiene el honor de ser considerada la primera enfermera profesional de América y fue esencial en el área de la salud mental al promover el cuidado del enfermo mental y crear escuelas para este fin $^{39}$. Para 1890, en Argentina se creó la primera escuela de enfermería de Latinoamérica, a la que siguieron países como Cuba, Chile y México ${ }^{40}$. La lucha intelectual no cesó y en 1907, la norteamericana Isabel Hampton logró incluir los estudios de enfermería en el ámbito universitario, los cuales iniciaron en la Universidad de Columbia (Nueva York) ${ }^{41}$. Gracias a estos avances, además de la emergencia de la enfermería técnica y la separación de lo político y religioso se gestó el camino hacia la profesionalización ${ }^{42}$. Esto se extendió al área de la salud pública y gracias a Lillian Wald se creó el primer sistema público de enfermería y con ello, la Organización Nacional de Enfermería de Salud Pública de la cual fue la primera presidenta ${ }^{43}$. Asimismo, creó lo que se conoció como Enfermería de Salud Pública, término que abandonó la Organización Mundial de la Salud en 1974 para acuñar el de Enfermería Comunitaria $^{44}$.

El desarrollo epistemológico ha sido clave para la enfermería y ha representado una oportunidad para figuras como Virginia Henderson, Hildegard Peplau y Dorothea $\mathrm{Orem}^{45}$, entre otras. Virginia Henderson, por ejemplo, fue trascendental al proponer niveles de relación entre la enfermera y el paciente, además de fomentar el hábito de la investigación ${ }^{46}$. Su trabajo trascendió al definir la función propia de la enfermera, concepto que en 1961 fue adoptado por el Consejo Internacional de Enfermería ${ }^{47}$. Esta función consiste en 
"atender al individuo, enfermo o sano, en la ejecución de aquellas actividades que contribuyen a su salud o a su restablecimiento (o a evitarle padecimientos en la hora de la muerte), actividades que él realizaría por sí mismo si tuviera la fuerza, voluntad o conocimiento necesarios. Igualmente corresponde a la enfermera cumplir esta misión en forma que ayude al enfermo a independizarse lo más rápidamente posible" ${ }^{\prime 47}$. En esta línea, Vera Fray estableció que una vez las necesidades del paciente son identificadas, el siguiente apartado es el Diagnóstico de Enfermería ${ }^{48}$. Bajo este concepto nació el Grupo Nacional para la Clasificación de Diagnósticos de Enfermería, en 1973 se promulgó la Clasificación de Diagnósticos de Enfermería y para 1982, se crea la North American Nursing Diagnosis Association (NANDA) ${ }^{49}$.

Es claro que los modelos teóricos han sido un eje de construcción sustancial para la enfermería. Y aunque son muchos los que han tenido gran repercusión, cabe mencionar algunos otros como el modelo de interacción de Martha Rogers, lo propuesto por Dorothy Johnson con el modelo de sistemas conductuales o el trabajo de Jacqueline Fawcett con el análisis y evaluación de modelos conceptuales de enfermería ${ }^{50}$. Uno de los de mayor renombre en las últimas décadas es el modelo de adaptación de Roy, el cual le significó a su autora Callista Roy junto con el trabajo de toda una vida, ser reconocida en 2007 como 'leyenda viviente' por la Academia Americana de Enfermería ${ }^{51}$. Ahora bien, más allá del aporte de cada uno de los diferentes modelos propuestos en un momento histórico puntual, lo que subyace en ellos es una reflexión del quehacer. Así, el pensar la profesión es producto de la experiencia diaria, y para la enfermería, tanto en lo práctico como en lo académico, su construcción depende del conocimiento enfermero ${ }^{52}$. Esa búsqueda de la creación de ciencia en torno a la enfermería ha promovido la investigación, el desarrollo de marcos conceptuales para la academia, y de una u otra forma ha llevado a la profesión a un nivel superior $^{52}$.

El presente y futuro de la enfermería se encaminan en lograr articular el trabajo entre la práctica diaria y la investigación ${ }^{53}$. Desde finales del siglo pasado, la investigación en torno al cuidado de enfermería ha cobrado protagonismo, aunque todavía constituye un campo por desarrollar ${ }^{54}$. De hecho, la investigación en enfermería constituye una dimensión esencial ya que cada vez más se requiere de la mejor evidencia para la toma de decisiones. Ahora bien, en el marco en un nuevo siglo y a la luz de nuevos Objetivos de Desarrollo del Milenio (ODM), la enfermería enfrenta nuevos retos.
El desarrollo de nuevas competencias ${ }^{55}$, por ejemplo, es un valor agregado que permitirá a la enfermería posicionarse como una pieza clave en el renacer de la Atención Primaria en Salud (APS). En la actualidad, en un mundo globalizado a merced de la tecnología, la comunión entre la enfermería y las Tecnologías de la Información y las Comunicaciones (TIC) es un camino emergente para desarrollar las potencialidades de una profesión que se reinventa y da forma a la enfermería del siglo XXI.

\section{CONCLUSIONES}

La historia evidencia lo cautivante que ha sido la enfermería desde sus inicios y la relevancia de las órdenes religiosas para lo que es hoy la profesión. Sin embargo, más allá del valor de lo histórico per se, el mensaje a resaltar es que las prácticas cuidadoras han estado enmarcadas por la tenacidad, la reinvención, la reflexión del quehacer y la capacidad para afrontar desafíos. Ahora bien, esos mismos obstáculos han estado embebidos por virtudes como: sacrificio, valor, disciplina, bondad, respeto y compasión, entre otros. Así, la enfermería no solo se ha edificado al dotarse de valores propios, sino que lo ha logrado al asistir al prójimo y con ello, ha contribuido a la construcción social. Hoy en día, la dinámica de un mundo que viaja de manera acelerada da cabida a nuevos horizontes para una profesión que tiene mucho más para aportar a la sociedad. En el contexto de la nueva dinámica de atención sanitaria que se enmarca en la eficacia, la eficiencia y la efectividad, la enfermería se convierte en una profesión que proyecta un futuro prometedor y con mayor capacidad de decisión y autonomía. La reinvención de la enfermería, entonces, se gesta a la luz de las tecnologías emergentes, los nuevos espacios sociales, los nuevos modelos teóricos, y las transiciones epidemiológicas y demográficas que en conjunto, potencian a esta profesión como actual y futura protagonista del complejo y dinámico campo de la salud.

\section{REFERENCIAS}

1. Matesanz MA. Pasado, presente y futuro de la Enfermería: una aptitud constante. Rev Adm Sanit. 2009; 7(2): 243-60.

2. Robinson V. White caps: the story of nursing. 1ra edición. Michigan: Lippincott, 1946: p. 113.

3. Sánchez Sendra AP. La historia de la enfermería en España y Latinoamérica. 1ra edición. España: Edición Kindle, 2014.

4. Siles J. Historia de la Enfermería. Madrid (España): 
Editorial Difusión Avances de Enfermería (DAE), 2011.

5. Monge M. Los cuidados enfermeros como estrategia de cambio económico y social en el Renacimiento Europeo. Rev Index Enferm. 2003; 12(43): 55-58.

6. Morales CT, García J. Hôtel-Dieu de París; orígenes y aparición de las primeras enfermeras religiosas de la historia. Rev Cult Cuidados. 2013: 35: 42-45.

7. Chevalier A. L'Hôtel-Dieu de Paris et les Soeurs Agustines. Un volum. París: Libraire de la Societe de l'Histoire de Paris; 1901.

8. Coyecque E. L'Hôtel-Dieu de Paris au Moyen Age. París: Ed. Daupeley-Gouverneur; 1891.

9. Nutting MA, Dock LL. A history or Nursing (The Evolution of Nursing Systems from the earliest times to the foundation of the first English and American training School of Nursing). New York: Putnam's Sons; 1907.

10. Aparicio T. Las Religiosas Agustinas del Hôtel-Dieu de París. Rev Arch Agustiniano. 1979; 63(181): 81108.

11. Castro FJ, Castro M, Megías F, Martín FV, Causapie A. Arquitectura hospitalaria y cuidados durante los siglos XV al XIX. Rev Cult Cuidados. 2012; 16(32): 38-46.

12. Morales C, García FJ. Hotel-Dieu de París: reformas materiales, estructurales y funcionales entre los siglos XII y XVIII. Rev Cult Cuidados. 2013; 17(36): 29-43.

13. Dubois G. Historia Ecelsiae Parisiensis. Societatem. París: Bibliopolarum Parisiensiu; 1710.

14. Salas PM. El reformismo social y sanitario de Concepción Arenal, una contribución a la identidad de la enfermería contemporánea. San Vicente (Alicante): Editorial Club Universitario; 2012.

15. Rivera MM. La diferencia sexual en la historia. Valladolid: Universitat de Valencia; 2005.

16. Ayguals W. El panteón universal: diccionario histórico. Madrid: Imprenta de Ayguals de Izco Hermanos; 1853.

17. Hernández JJ. Enfermedad y muerte en América y Andalucía (Siglos XVI-XX). Madrid (España): Editorial CSIC-CSIC Press; 2004.

18. Sacchetti L, Rovere M. Surgimiento de la Enfermería Moderna; Descifrando Mitos, Contextos y Devenires. En: Jornadas Nacionales de Debate Interdisciplinario en Salud y Población. Buenos Aires: Asociación Civil El Ágora; 2009.

19. García MJ, García AC. Andrés Fernández, un enfermero onubense, su papel en la reforma de la enfermería hospitalaria española del siglo XVII. Huelva en su historia; 1997.

20. García Martínez AC, García Martínez MJ,
Hernández Martín F, Pérez Melero A, Pinar García ME. Aproximación a la enfermería Española de los Siglos XVI-XVII. Index Enferm. 1998; 20-21: 5455.

21. García MJ, García AC. El manual Instrucción de enfermeros (1625), compuesto por los enfermeros obregones, y los cuidados urológicos en los hospitales del siglo XVII. ENFURO. 2012; 122(2): 4-10.

22. León J. Textos de enfermería impresos en España durante los siglos XVI y XVII disponibles en bibliotecas digitales. Enfermería Global 2013; 32: 280-287.

23. Zambrano MS. Técnicas básicas de enfermería. Madrid (España): Ediciones Akal, S.A; 2003.

24. Hernández N, Chiricó S. Ana Packer: construyendo el saber y hacer enfermero: de Inglaterra a CuñapirúCorrales, 1841-1930. Montevideo (Uruguay): Ediciones Trilce; 2004.

25. Donahue P. Historia de la enfermería. Barcelona (España): Ediciones Doyma; 1985.

26. Helmstadter C, Godden J. Nursing before Nightingale. 1815-1899. England: Ashgate Publishing Limited; 2011.

27. Arias J, Ángeles M, Arias JI, Lorente L. Generalidades médico-quirúrgicas. Madrid (España): Editorial Tébar; 2001.

28. Rodríguez R. Una Obra de Arte; la esencia del arte y la medicina. Bogotá (Colombia): Editorial Publimpacto; 2010.

29. Antón MV. Las enfermeras, entre el desafío y la rutina: una mirada al siglo XXI. Madrid (España): Ediciones Díaz de Santos; 1998.

30. Roux G, Halstead J. Issues and Trends in Nursing: essential knowledge for today and tomorrow. United States of America: Jones \& Bartlett Publishers; 2009.

31. Nightingale F. Notas sobre enfermería: qué es y que no es. Elsevier España; 1990.

32. Rodríguez ME, Hernández S. Breves aspectos de la enfermería según Florence Nightingale. Enf Neurol. 2013; 12 (1): 45-47.

33. Directrices para la enseñanza de enfermería en la educación superior. Bogotá, (D.C.): Asociación Colombiana de Facultades de Enfermería; 2006.

34. Zambrano GE. Ethel Fenwick una mujer del siglo XXI en el siglo XIX. Investigación en Enfermería: Imagen y desarrollo. 2003; 5(1): 79-85.

35. Bernalte A., Miret MT. Manual de enfermería comunitaria.. Libros en Red; 2005.

36. Suárez M. Más allá del Homo Sapiens-Volumen II. Victoria (Canadá): Trafford Publishing; 2000.

37. Oguisso T. La incidencia del Consejo Internacional 
de Enfermeras en la historia y socialización de la enfermería. Revista Cultura de los Cuidados. 2012; 16(32):23-37.

38. Martín A. Patología quirúrgica. Madrid (España): Elsevier España2004.

39. Esguerra I. Rol del profesional de enfermería en salud mental y psiquiatría. Av Enferm. 1991; 9(1): 27-34.

40. Verderese O. Análisis de la enfermería en la América Latina. Educ Méd Salud. 1979; 13 (4): 315-340.

41. Espejo MD. Retos bioéticos de la enfermería actual. España: Fundación Bioética; 2008.

42. García C, Martínez ML. Historia de la enfermería: evolución histórica del cuidado enfermero. Madrid (España): Elsevier; 2001.

43. Chiriboga PP. Lillian Wald, la coyuntura antisegregacionista. Pub Médicas. 2011; 1(1): 96-98.

44. Alonso O. La especialidad en Enfermería familiar y comunitaria. Volver a empezar. Rev Seapa. 2013; 11: 27-30.

45. Hernández F, Lastra R, Alcaraz S, González JM. La enfermería en la historia. Un análisis desde la perspectiva profesional. Rev Cult Cuidados. 1997; 2: 21-35.

46. García M. El proceso de la enfermería y el modelo de Virginia Henderson. $2^{\circ}$ Edición. México, (D.F.): Editorial Progreso; 2004.

47. Organización Panamericana de la Salud. Principios básicos de los cuidados de enfermería. Washington, (D.C.): Organización Panamericana de la Salud; 1961.

48. Bello N. Fundamentos de enfermería. Ciudad de la Habana (Cuba): Editorial Ciencias Médicas; 2006.

49. Guirao-Goris JA, Camaño RE, Zambrano AC. Diagnóstico de Enfermería. Categorías, formulación e investigación. Ediversitas; 2001.

50. Cisneros F. Introducción a los modelos y teorías de enfermería. Popayán (Colombia): Universidad del Cauca; 2002.

51. American Academy of Nursing. Washington, (D.C.): American Academy of Nursing; 2014.

52. Marriner A, Raile M. Modelos y teorías en enfermería. Sexta Edición. Barcelona (España): Elsevier España; 2008.

53. Parrado YM. Investigación, columna para la práctica de enfermería. Rev Av Enferm. 2010; 28(1): 11-12.

54. Gallegos E. Evaluemos la práctica social de la enfermería. Invest Educ Enferm. 2006; 24(2): 13-14.

55. Jara P, Stiepovic J. Currículo por competencias en el postgrado de enfermería. Invest Educ Enferm. 2007; 25 (2): 122-129. 